\title{
The Whole Earth Telescope Network's August 2008 Workshop
}

\author{
H. L. Shipman \\ Delaware Asteroseismic Research Center, Mt. Cuba Observatory and University of \\ Delaware, Newark, DE 19716 USA
}

\section{Introduction}

The Whole Earth Telescope project hosted a meeting in August 2008 in Newark, Delaware. The purpose of this meeting was to review recent progress in the field, welcome new participants to the network, introduce new and old participants to the way that the new network functions, discuss how we operate, and make plans for the future. This paper summarizes the workshop as a whole. The following papers in this issue describe particular areas of progress.

A fair number of groups have put together networks of people and telescopes to concentrate on particular stars or particular areas of astronomy. Most of these networks have lasted for a few years and then fallen into disuse when the original project driving them was completed. In the field of variable stars, two networks have lasted for much longer: the delta Scuti network established by Michel Breger in the early 1980s and the Whole Earth Telescope, which had its beginnings in the late 1980s under the leadership of Ed Nather and Don Winget. Both of these networks still exist and are doing good science. See DARC (2007) for a more extended history of DARC and DSN (2005) for information on the Delta Scuti Network.

Starting in the early 1990s, the history of WET is described in a series of conference proceedings. The first WET workshop was held in Austin, Texas, in November 1991, and as far as I know there are no formal conference proceedings. There are proceedings for the second workshop (Meistas \& Solheim 1993) held in Moletai, Lithuania; the third (Meistas \& Solheim 1995), held in Ames, lowa; the fourth (Meistas \& Moskalik 1998), held in 1997 in Koninki, Poland; the fifth (Meistas \& Vauclair 2000) held in 1999 in Bonas Castle, France, under NATO auspices.

The summer 2007 meeting was also the first meeting held since the relatively recent management change of the Whole Earth Telescope (WET) project. 
In 2004, a variety of circumstances including funding difficulties led the WET leadership to encourage Judi Provencal and me to seek an alternative source of funding for WET. We obtained a major grant from the Crystal Trust. As a result, the Mt. Cuba Astronomical Observatory and the University of Delaware jointly established the Delaware Asteroseismic Research Center (DARC) which oversees a number of variable star projects. WET is by far the largest project running under the auspices of DARC.

This paper and the papers which follow in this special section of Communications on Asteroseismology is intended to give the flavor of the summer 2007 WET workshop. We decided not to make the effort to gather a "complete set of papers or to somehow include every comment or part of the discussion. Rather, we invited every workshop participant to send us a contribution if they wished. These papers can serve to give present and future readers of this journal a flavor for where the WET project is in 2008.

\section{GD 358 and Testing Stellar Convection}

An important paper published in the fall of 2004 stimulated an important and new line of thinking, one which has guided much of WET planning for the next few years. Mike Montgomery (2004) suggested that the nonlinearities in stellar light curves could be used as a way to test convection. Convection has been on various lists of unsolved problems in astrophysics (and physics) for several decades. We all use variants of the classical mixing-length theory because, really, there's nothing else sensible to do. Until Montgomery published these ideas in 2004, the only tests of the mixing-length theory were rather indirect.

The Montgomery scheme is conceptually quite simple and elegant. Pick a star whose pulsation spectrum is sufficiently complex that the pulsations are nonlinear, but sufficiently simple that a reasonable set of observations can permit its pulsation modes to be identified securely. The amplitude pattern of the pulsations will be established by the way that the star responds to these pulsations, the way that its temperature stratification changes in response to periodic changes in the total energy flow. The star's light curve will depend on how convection responds to the driving provided by the pulsations. Compare the actual light curve with models and you can test convection.

This challenge is ideally suited to the combination of telescopes which are currently available for WET. The WET network contains a large number of small telescopes where time is readily available. If a star is bright enough to be accessible to these telescopes, they can identify the pulsation modes. At the same time, a larger telescope (such as a $2-\mathrm{m}$ or 4-m, or possibly even larger) can provide a light curve with high signal/noise, typically $S / N>100$, to test various theories of convection. 
With these considerations in mind, we began to plan a WET run which eventually took place in May 2006. XCOV25 became the WET run which included one of the largest number of telescopes in the history of WET. ${ }^{1}$ While our efforts to obtain data from really large telescopes such as the Hawaii $10-\mathrm{m}$ and SOAR were not successful, 12 of the 20 telescopes involved in WET were 2-m or larger and thus were able to obtain the kind of detailed light curves that were needed to implement the Montgomery test.

The results from this WET run were presented a year after the run at two conferences (the white dwarf conference in Leicester and the variable star conference in Vienna), and in some papers in this volume. Nevertheless, the papers presented in the summer of 2006, a few months after the observations were obtained, presented much more than a simple look at the power spectrum. Those papers (see Provencal et al. 2007a, 2007b) demonstrate that the Montgomery test works, that the mixing length theory with a reasonable choice of parameters does indeed produce an adequate fit to the light curve. It has encouraged us to widen our search for more stars like GD 358 so that we can extend the Montgomery test. "Like GD 358" does not necessarily mean DBV pulsators; rather, it means stars of similar brightness, and similar types of pulsation spectra: complex enough to be nonlinear but not so complex as to make the analysis impossible.

The data set for GD 358 is extraordinarily rich, and as time goes on our interpretation becomes progressively more detailed. The papers presented in the summer of 2006 at the biennial white dwarf conference and the Vienna variable star conference (Provencal et al. 2007a, 2007b) contain much deeper interpretations of new kinds of phenomena than was the case before. There is yet more in the papers published in this journal. The final papers will be deeper still.

\section{New Ways of Operating WET}

With the re-establishment of the WET under Delaware auspices, we made some changes in the way it works. Some were dictated by the completion of the transition from a network of telescopes using the same Texas three-star photometers to a network of telescopes and CCD cameras. Some reflected the choices of the new leadership. Considerable amounts of time at the conference were spent discussing how well these choices had worked and how they could be improved.

The role of headquarters. In the past, a WET run always had a headquarters, because simply implementing the run was often more complex

\footnotetext{
${ }^{1} \mathrm{XCOV}$ stands for extended coverage, and the number is the number of the WET run. A list of WET runs is given at the DARC website at http://www.physics.udel.edu /darc/wet/campaigns.htm.
} 
than was the case for XCOV25. A small change in the technology which had surprisingly large implications was the availability of signals from atomic clocks over the internet. We no longer needed phone calls between sites in order to establish a uniform time base. As a result, emails sufficed in almost all cases to find out whether and when a particular site could observe, and when it could observe. Because there was only one target, no decisions had to be made.

Supporting Software. Headquarters became a data analysis center. Antonio Kanaan, who had developed a number of scripts which allowed us to use the power of IRAF to analyze large volumes of data quickly, led an effort to analyze the real data quickly. Judi Provencal, Andrezj Baran, and Mike Montgomery also participated in this effort. Even though there were about 100,000 images which comprised the final data set, the final light curve was basically in place at the end of the WET run. Data reduction has never been so speedy within WET. It has often been the choke point which has delayed the publication and analysis of data.

There was considerable discussion at the summer meeting regarding how we should handle the question of providing appropriate support to individual observers in order to make data acquisition and data reduction as easy as possible. Many telescopes and observers have their own approach to data acquisition; the only additional difficulty with WET is being sure to provide the appropriate information, especially timing, for those at headquarters. Then it is up to those at headquarters to use their favorite software for data analysis, which at the moment (early 2008) appears to be Antonio Kanaan's scripts. Delaware graduate student James Dalessio discussed some software he is developing which may make it easier to analyze data from stars with close companions and from sites where the image of the target star shifts position during the night. Reed Riddle talked about the capabilities of the XQED software package which had been used in XCOV23-24.

Transition to an all-CCD network. When WET was originally established, the technology of CCDs was relatively new and observing practices were changing rapidly. One of the founding principles of WET was that every telescope would have the same instrumentation. Now that CCDs are sufficiently well established, it has become neither practical nor sensible to insist that all sites use a standard instrument such as the Texas 3-star photometer. Prior to XCOV25, there was much discussion over the internet about what we should do. Many sites on XCOV25 used a specific instrument, namely a back-illuminated camera provided by Apogee. However, the use of Apogees was not universal. It has seemed that the different back-illuminated cameras are similar enough so that our data analysis for XCOV25 has worked.

Other issues. Scot Kleinman described his use of the PUBLISH software package which makes it possible to post light curves quickly to the internet site. 
This will be tried in forthcoming WET runs and campaigns. The sense of the meeting was that failure to get light curves publicly available quickly was one important thing which should be improved after XCOV25.

After the meeting, it was clear that it was important to have periodic meetings such as this workshop. Issues large and small need to be discussed so that the Delaware group can take action to implement improvements. While email conversations and special get-togethers at meetings like the variable star meetings and the biennial white dwarf workshops are helpful, it seems that WET workshops are another important way that the WET team can move forward. The WET management council, known more informally as the Council of the Wise or COW, gave its approval to the way that the WET is being run, but made a strong suggestion that the bandwidth of the internet connection to the Mt. Cuba Observatory, the site of headquarters, be improved.

\section{WET Science and Future WET Targets}

The final selection of WET targets is done, as was done in the past, by the WET director (currently Judi Provencal), consulting with the COW and after considerable exchange of e-mails. However, one of the things that can be accomplished at a WET workshop can be a great deal of face-to-face discussion of the merits of various targets which were proposed for the next WET run, which is scheduled for April 2008. About half of the time at this workshop was devoted to such discussion.

It is probably not worth recording the details of this discussion in an overview of the workshop here. Target selection was made when most of this paper was written. Final editing of this paper took place after the April 2008 WET run took place, and this authors preliminary assessment is that the spring 2008 WET run, XCOV26, was a success. However, several points emerged from the discussion which are worth recording for wider circulation.

Kepler reviewed some results of his group and of the Texas group which have led to a considerable increase in the number of known variable white dwarf stars. He reported a number of 140 , with 80 of them discovered as a result of the Sloan Digital Sky Survey. Unfortunately many of the newly discovered white dwarf variables are very faint. The dynamics of time availability on telescopes of various sizes is very much in flux right now, and one of the issues for the future of this field will be a changing definition of what is too faint for the community to work on. Kawaler pointed out that there is a senior review at the NSF of time-domain astrophysics going on as of the fall of 2007.

A novel concept which emerged from the discussion was the idea of designating one or more stars as targets for observation before such a star becomes a target for a full fledged WET run, where the 20-plus telescopes in the WET 
community are all brought to bear on one single target, where several observers are sent to remote places to operate telescopes, and where there is a full-fledged headquarters. Such precursor observations were actually done in calendar year 2007 for two stars: WD1524-0030 and G38-29. G38-29 is an example of a star where very little data existed beyond the discovery data from decades ago (1977).

After the workshop, WET director Provencal announced that there would be a mini-campaign on G38-29 in early November 2007. This almost became a WET run, though a tentative decision was made not to give it an XCOV designation. We did not have a "headquarters but Antonio Kanaan did come from Brazil to analyze the data. We did make the effort to send two observers to Hawaii. We obtained some 2-m observations of this star.

There was some discussion of future missions and large telescopes which might affect the WET community. Spacecraft like COROT and KEPLER will provide information on variable stars. These missions are often designed with brighter variables in mind, and time will tell how the white dwarf community can best take advantage of the capabilities given by these missions. The science working group of the ground-based LSST has contacted the white dwarf community for input, and again there will be some efforts to maintain contact.

We also heard some presentations from and about new partners. Xiaojun Jiang of the National Astronomical Observatory of China presented the capabilities of the observatories which currently exist in China, including both 1-m and 2-m telescopes. There are plans for additional telescopes in western China which may be extremely useful for WET science. He and his colleagues have been very active contributors to WET. Hari Om Vats presented a report on behalf of a new observational astronomy group at the Mt. Abu Observatory, near Gurushikhar, the highest peak in the whole of central India. They have a $1-\mathrm{m}$ class telescope with a very good camera. This group participated in the November campaign on G 38-29. I presented a report on his negotiations with the Las Cumbres Observatory, which is planning to build a worldwide network of telescopes in the 1-2 meter range (see www.lcogt.net for details). While their primary purpose is observing planetary transits and gravitational lensing, they are also open to collaborations such as WET. Las Cumbres participated in the November campaign and the April WET run.

The community of astronomers who studies white dwarf stars has a tradition at its meetings, which is enthusiastically followed both at the meetings of the whole white dwarf community (which occur in even-numbered years) and in WET workshops. We have tours and we have very nice conference dinners. Science does get discussed at these tours, of course. WET projects coordinator Teresa Holton arranged an excellent tour of the nearby Winterthur house and gardens, which present the collections of P.S. DuPont, a man who assembled 
rooms of furniture to represent particular periods in American history. She also arranged an excellent dinner at Harry's Savoy Grill, a nearby restaurant which did an excellent job in presenting seafood characteristic of the Chesapeake Bay and parts of the coast which lie more to the northeast. We were pleased to host some of our donors at this dinner. Without the support of the Crystal Trust for the DARC and WET projects, none of this would have happened. The author of this paper also acknowledges support from the National Science Foundations Distinguished Teaching Scholars program.

\section{References}

DARC 2007. http://www.physics.udel.edu/darc/darc_history.html (Accessed January 14, 2008)

DSN 2005. http://www.univie.ac.at/tops/dsn/intro.html (Accessed April 24, 2008)

Meistas, E. G., \& Solheim, J. E. 1993, Proceedings of the Second WET Workshop, BaltA, 2, 360

Meistas, E. G., \& Solheim, J. E. 1995, Proceedings of the Third WET Workshop, BaltA, 4, 105

Meistas, E. G., \& Moskalik, P. 1998, Proceedings of the Fourth WET Workshop, BaltA, 7, 1

Meistas, E. G., \& Vauclair, G. 2000, Proceedings of the Fifth WET Workshop, BaltA, 9, 1

Montgomery. M. H. 2005, AJ, 633, 1142

Provencal, J., Shipman, H. L., \& the WET Team 2007a, CoAst, 150, 293

Provencal, J. L., Shipman, H. L., Montgomery, M. H., et al. 2007b, 5th European Workshop on White Dwarfs ASP Conference Series, ASPC, 372, 623

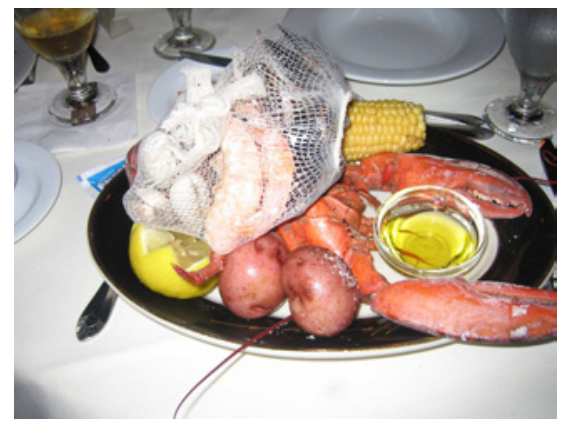

Delicious seafood dinner at Harry's Savoy Grill. 


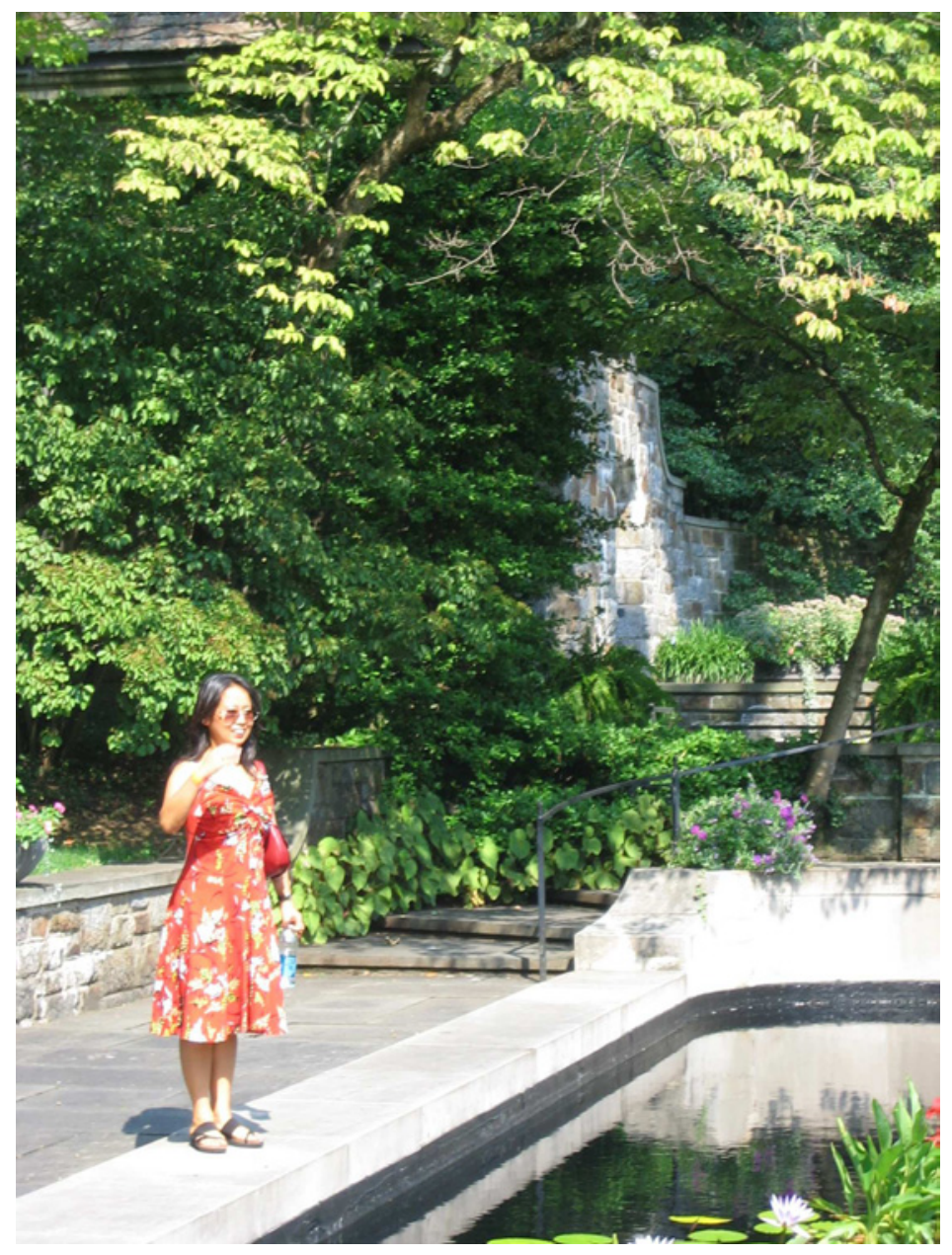

A. Nitta at Winterthur Gardens. 